\title{
Enhanced Detection of Sulfated Glycosylation Sites in Glycoproteins
}

\author{
Hui Jiang, Janet Irungu, and Heather Desaire \\ Department of Chemistry, University of Kansas, Lawrence, Kansas, USA
}

We demonstrate a method that enhances the mass spectral signal of mono- and disulfated glycopeptides, present in glycoproteins that contain many other nonsulfated glycoforms. This method utilizes the tripeptide Lys-Lys-Lys as an ion-pairing reagent to complex selectively to sulfated species, and enhance their ion signal. The method is applied to the analysis of glycopeptides released from the enzymatic digestion of ovine luteinizing hormone. In this analysis, a disulfated glycopeptide is identified that was previously not detectable by MS assays, and a monosulfated glycoform, present at less than $1 \%$ abundance, is identified without any separation or enrichment of these species prior to analysis. In addition to enhancing the ion signal of sulfated glycopeptides, the ion-pairing technique is useful in obtaining structural information about the sulfated species. (J Am Soc Mass Spectrom 2005, 16, 340-348) (c) 2005 American Society for Mass Spectrometry

S ulfation is a functionally significant biological modification of carbohydrates. It is an integral, ubiquitous component of the polymeric glycosaminoglycans, present in the extra-cellular matrix [1]. Changes in glycosaminoglycan sulfation have been linked to arthritis and other disease states [2-4]. Sulfation also is prevalent on cell-surface oligosaccharides in virtually all mammalian tissues, and its presence is fundamental in cellular communication events [5]. Additionally, sulfation is a common modification of glycoproteins, including erythropoeitin [6], mucins [7, 8], and the gonadotropin hormones, lutropin, follitropin, and thyrotropin [9]. When present in glycoproteins, sulfation can alter biological recognition $[10,11]$ and/or facilitate rapid clearance of the protein from the body [12]. Because sulfation is a pervasive, functionally significant biological modification, analytical methods that can monitor its presence are required to better understand its role in protein function, in cell signaling events, and in diseases where sulfation patterns are altered, like arthritis $[2,3]$ and cystic fibrosis $[5,7,8]$.

While sensitive and selective detection of sulfated compounds is of paramount importance, research in this field is restricted by the fact that sulfated carbohydrates are not trivial to detect and characterize [13], especially when they are present in complex matrices. Metabolic radiolabeling is one strategy that is commonly used to detect sulfated carbohydrates $[5,9,11$, 14, 15]. Radiolabeling, while hazardous and laborintensive, is quite useful because sulfated glycoforms are selectively labeled and detected, in the presence of

Published online January 13, 2005

Address reprint requests to Dr. H. Desaire, Department of Chemistry, University of Kansas, 1251 Wescoe Hall Drive, Lawrence, KS 66045, USA. E-mail: hdesaire@ku.edu many other endogenous compounds. Mass spectrometry, which affords more rapid analysis times, could obviate the need for radiolabeling in some cases, if sulfated glycoforms could be detected in complex matrices.

Recent experiments using LC-MS (with ionization in the negative ion mode) have shown that sulfated carbohydrates can be separated from other endogenous species and detected by MS methods [6-8, 13]. The application of this detection method, ESI-MS in negative mode, is not straightforward for the analysis of sulfated glycopeptides; this is demonstrated herein. In addition to the problems of detecting glycopeptides in negative ESI, it is well known that in positive ion mode the signal of sulfated compounds is suppressed by mass spectral signals of nonsulfated compounds. We have demonstrated this recently in the analysis of sulfated glycopeptides released from luteinizing hormone [16]. In essence, the detection of (underivatized) sulfated glycopeptides is not ideal in negative mode or positive mode, and complementary detection methods are required to improve the detection and characterization of these compounds.

Here, we demonstrate a method that can enhance the signal of mono- and disulfated glycopeptides, in the presence of nonsulfated glycoforms, facilitating their detection in positive ion ESI-MS. The method utilizes ion-pairing reagents that selectively bind to sulfated carbohydrates and increase their mass spectral signal. Similar ion-pairing strategies were used by other researchers to ionize highly sulfated carbohydrates by MALDI [17], or by ESI [18-20]. But in these previous examples, ion-pairing was used for purified, highly sulfated, carbohydrates. In the study presented here, new, low molecular-weight ion-pairing agents are identified that selectively enhance the signal of mono- and disulfated glycopeptides, present in highly complex mixtures. The 
method is utilized to detect several sulfated glycopeptides that are present in low-abundance in the glycoprotein, ovine luteinizing hormone. The ion-pairing technique is superior to using negative ion ESI-MS because MS/MS of the ion-pair complexes can be used to confirm the composition and structure of the sulfated glycopeptides; while MS/MS data from the negatively ionized glycopeptides is ineffective at confirming the composition or the structure of the ions of interest.

\section{Experimental}

\section{Sample Preparation}

The peptides, RSK and RGK, were synthesized by the University of Kansas Biological Research Service Laboratory, using solid-phase peptide synthesis. All other reagents were purchased from Sigma-Aldrich (St. Louis, MO) and used without further purification. Stock solutions of all the peptides were prepared, at a concentration of $1.4^{*} 10^{-3} \mathrm{M}$. The solvent in all cases was $\mathrm{MeOH}: \mathrm{H}_{2} \mathrm{O}$ (1:1) containing 0.5\% acetic acid. Stock solutions of the carbohydrates were prepared in this solvent at a concentration of $1.82^{*} 10^{-4} \mathrm{M}$. The internal standard, caffeine, was also present in this solution, at a concentration of $1.8^{*} 10^{-3}$ M. Just prior to analysis, ion-pair complexes of the carbohydrates and peptides were formed by combining $10 \mu \mathrm{L}$ of peptide solution with $10 \mu \mathrm{L}$ of carbohydrate solution, vortexing, and injecting $1 \mu \mathrm{L}$ of this mixture into the mass spectrometer.

Glycopeptides from ovine luteinizing hormone, $\alpha$ subunit, were generated in the laboratory of Dr. George Bousfield, Wichita State University, as described previously [16]. Briefly, after proteinase K digestion the released glycopeptides were isolated using centrifugal ultrafiltration and desalted. The dried product contained a mixture of peptides (verified by Edman sequencing) with the predominant species being the glycopeptide: N-I-T, glycosylated at the asparagine. The glycosylation was quite variable; it included sulfated and nonsulfated glycoforms. In all, about 20 different glycoforms were present in the mixture, based on mass spectrometric analysis [16]. For ion-pairing experiments, this sample, referred to henceforth as "the sulfated glycopeptide mixture", was combined with the ion-pairing reagent, Lys-LysLys. For analysis using the quadrupole ion trap mass spectrometer, the optimal sample concentration was a sulfated glycopeptide mixture, $1 \mu \mathrm{g}$, dissolved in 3 $\mu \mathrm{L}$ of a $1.25^{*} 10^{-3} \mathrm{M}$ solution of the ion-pairing agent. For analysis on the qTOF mass spectrometer, the optimal sample concentration was sulfated glycopeptide mixture at a concentration of $0.62 \mu \mathrm{g} / \mu \mathrm{L}$, in a $1.35^{*} 10^{-3} \mathrm{M}$ solution of the ion-pairing agent. This solution also contained an internal standard, hexa- $\mathrm{N}$ acetylchitohexaose, at a concentration of $2.5^{*} 10^{-5} \mathrm{M}$, for high-resolution mass measurements.

\section{Mass Spectrometry}

Low-resolution mass spectra were acquired on a Thermo Finnigan LCQ Advantage quadrupole ion trap mass spectrometer (San Jose, CA). Samples were introduced into the mass spectrometer by direct injection of 1-3 $\mu \mathrm{L}$ of sample into a mobile phase of $\mathrm{MeOH}: \mathrm{H}_{2} \mathrm{O}$ (1:1) containing $0.5 \%$ acetic acid. A Surveyor MS-Pump (Thermo Finnigan) was used to deliver the mobile phase at a flow rate of $10 \mu \mathrm{L} / \mathrm{min}$. Electrospray ionization, in the positive ion mode, was achieved using a spray voltage of approximately $4.0 \mathrm{kV}$. Nitrogen was used as a nebulizing gas, at 20 psi. Data were acquired and processed using Xcalibur 1.3 software (ThermoFinnigan, San Jose, CA).

High resoltuion mass spectra were acquired on a Micromass Q-TOF ${ }^{2}$ mass spectrometer, using nanospray. Each sample (about $10 \mu \mathrm{L}$ ) was loaded into a PicoTip (New Objective, Woburn, MA) and the PicoTip was mounted to the nanospray stack. A voltage of 700 to $1500 \mathrm{~V}$ was applied between the tip and the mass spectrometer inlet. Cone voltage was $40 \mathrm{~V}$. Accurate mass measurement was done with the addition of the internal standard hexa- $N$-acetylchitohexaose. Spectra were recorded in the positive ion mode.

\section{Results and Discussion}

A small library was acquired containing peptides that could potentially be used as ion-pairing reagents for sulfated carbohydrates and glycopeptides; see Figure 1. Large peptides (1000-2000+ Da) have been shown previously to coordinate to sulfated carbohydrates [17, 18]; but ion-pairing reagents with lower molecular weights are advantageous $[19,20]$. Each potential ionpairing agent has at least one Lys or Arg residue in its structure, which is important because these amino acids had been shown to complex to sulfated carbohydrates previously $[17,18]$. While non peptidic amines have also been used for ion-pairing with sulfated carbohydrates [19, 20], preliminary studies of possible nonpeptidic complexing agents indicated that aliphatic amines displayed lower binding affinity for monosulfated carbohydrates than several of the peptides herein.

To determine which ion-pairing reagents would effectively complex to sulfated glycans, a study was performed using the library of potential ion-pairing agents and four different carbohydrates, displayed in Figure 2. Three of these standards are different types of monosulfated carbohydrates, and one is disulfated. Four different standards were chosen because it is important that the ionpairing reagent bind to a variety of different types of sulfated carbohydrates. For each carbohydrate/peptide combination, a solution containing a 1:7 mole ratio of carbohydrate to peptide was injected into the mass spectrometer, and the ion-pair complexes present in the mass spectrum were identified. (The peptide is in excess because previous reports demonstrate this facilitates detection of the carbohydrates [18].) The complexing efficiency 


\begin{tabular}{|c|c|}
\hline Amino acids: & Lys, Arg \\
\hline Dipeptides: & $\begin{array}{l}\text { Lys-Lys (Abbreviated as } 2 \mathrm{~K} \text { ) } \\
\text { Lys-Leu (Abbreviated as KL) } \\
\text { Arg-Phe (Abbreviated as RF) }\end{array}$ \\
\hline Tripeptides: & $\begin{array}{l}\text { Lys-Tyr-Lys (KYK) } \\
\text { Arg-Ser-Lys (RSK) } \\
\text { Arg-Gly-Lys (RGK) } \\
\text { Lys-Lys-Lys (3K) }\end{array}$ \\
\hline $\begin{array}{l}\text { Tetrapeptide: } \\
\text { Larger peptide } \\
\text { Lys-Arg- } \\
\text { Ser-Arg- } \\
\text { Arg-Lys- }\end{array}$ & 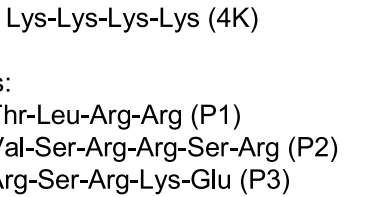 \\
\hline
\end{tabular}

Figure 1. Peptides and amino acids tested as potential ionpairing reagents.

of each of the peptides was evaluated, for each of the four carbohydrates.

In most cases, the peptides bind to the sulfated carbohydrates and form ion-pair complexes. Generally, when this occurs, the signal of the ion-pair complex is stronger than the signal of the sulfated carbohydrate, when no ion-pairing agent is present. One example of this is shown in Figure 3. Figure 3a contains the mass spectrum of just the sulfated monosaccharide GalNAc4S. Without the ion-pairing reagent, the sulfated monosaccharide appears in clusters containing various numbers of carbohydrates and sodium ions. Figure $3 \mathrm{~b}$ is this monosaccharide (at half the concentration), with the peptide Lys-Lys-Lys added. Once the ion-pairing reagent is added, no free sulfated monosaccharide is apparent in any form, including $\mathrm{m} / \mathrm{z} 346[\mathrm{M}+$ $2 \mathrm{Na}]+, 669[2 \mathrm{M}+3 \mathrm{Na}]+$, or $991[3 \mathrm{M}+4 \mathrm{Na}]+$. The monosaccharide is only observable as a complex with the peptide. The signal for the ion pair complex, $\mathrm{m} / \mathrm{z}$ 704, in Figure $3 \mathrm{~b}$ is 19 times greater than the signal for the most abundant ion in Figure 3a.

The mass of the ion, $m / z 704$, is consistent with the expected mass of the ion-pair complex [Lys-Lys-Lys

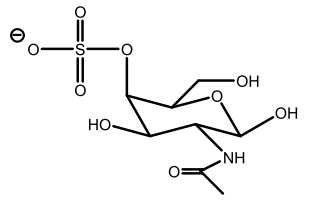

GalNAc 4S

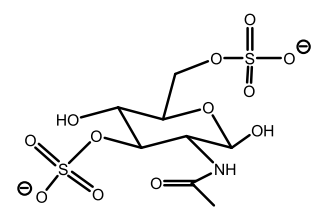

GIcNAc 3,6S

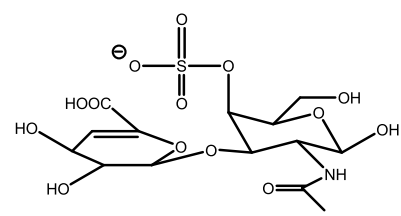

$\Delta$ di-6S

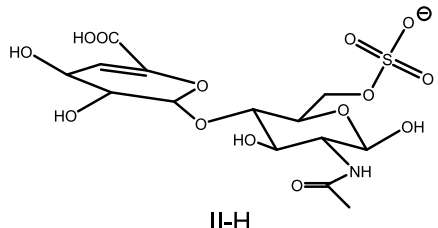

$\mathrm{II}-\mathrm{H}$
Figure 2. Sulfated carbohydrate standards used for ion-pairing experiments.
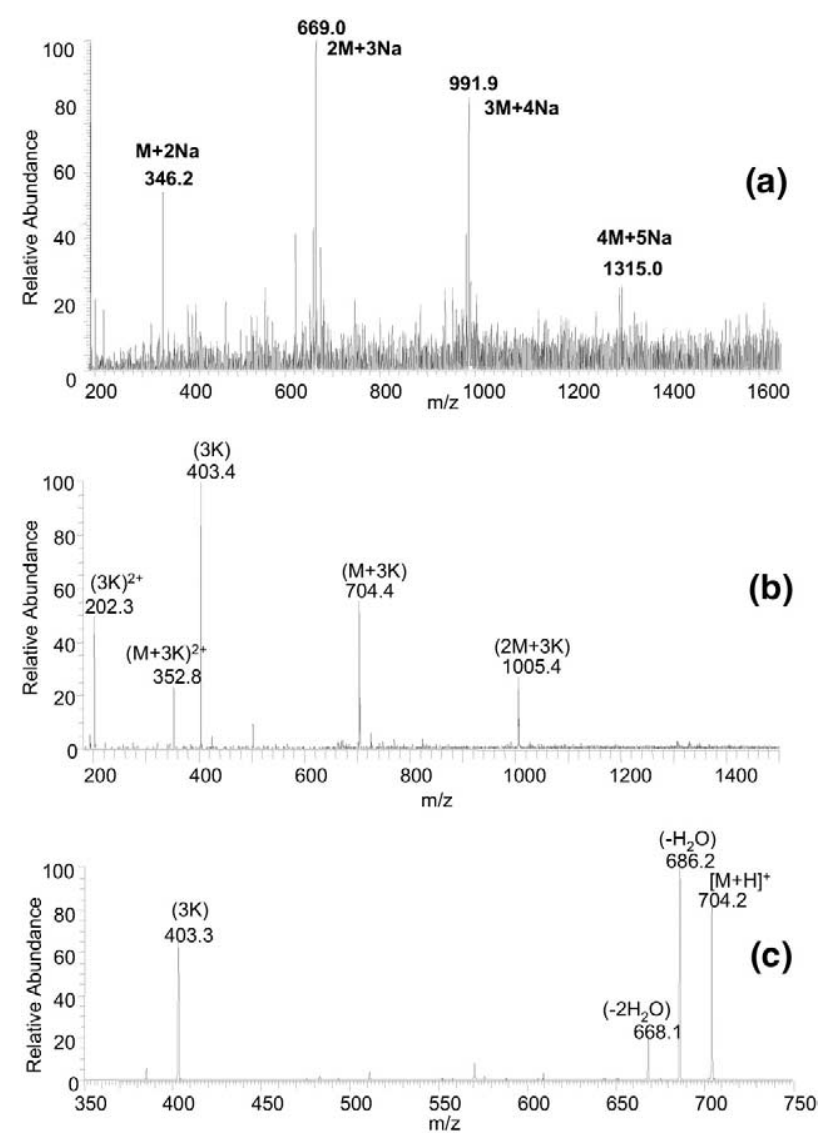

Figure 3. (a) MS data for the sulfated carbohydrate GalNAc4S, prior to addition of the ion-pairing reagent. (b) MS data for the sulfated carbohydrate, GalNAc4S, after the ion-pairing reagent Lys-Lys-Lys (3K) is added. (c) MS/MS data for the ion pair complex [GalNAc4S $+3 \mathrm{~K}]^{+}$.

+ GalNAc4S $]^{+}$. To provide additional confirmation that this ion corresponds to the expected ion-pair complex, an MS/MS experiment was performed on this ion, $\mathrm{m} / \mathrm{z}$ 704; see Figure 3c. This spectrum contains a product ion corresponding to [Lys-LysLys $+\mathrm{H}]+$, at $m / z 403$, along with ions corresponding to losses of water, which are typically observed during monosaccharide fragmentation [21, 22]. Thus, the MS/MS data supports the initial assignment, that the ion $m / z 704$ is an ion-pair complex containing the carbohydrate GalNAc4S, and the peptide Lys-LysLys.

To semiquantitatively determine which peptides had strong complexation efficiencies with the carbohydrates, the signal of the most abundant ion-pair complex was determined, relative to the signal of the largest peak corresponding to the uncomplexed peptide; see Table 1. For example, in Figure $3 b$, the ion at $\mathrm{m} / \mathrm{z} 704$ is at $55 \%$ relative abundance, compared to the signal for the ion $\mathrm{m} / \mathrm{z} 403$, which is the protonated peptide. The value " 55 " is reported for this carbohydrate/peptide pair in Table 1. For compounds that were identifiable in various charge states, only the peak with the highest abundance was chosen for comparison. 
Table 1. Relative complexation affinity for peptides*

\begin{tabular}{|c|c|c|c|c|c|c|c|c|}
\hline \multirow[b]{2}{*}{ Sulfated carbohydrate } & & \multicolumn{2}{|c|}{$\longleftarrow$} & \multicolumn{2}{|c|}{ Abundant ion-pair complexes } & \multicolumn{3}{|c|}{$\longrightarrow$} \\
\hline & & $2 \mathrm{~K}$ & \multicolumn{2}{|l|}{$3 \mathrm{~K}$} & $\mathrm{P} 2$ & \multicolumn{2}{|c|}{ P3 } & $\mathrm{P} 1$ \\
\hline GalNAc 4S & & 56 & \multicolumn{2}{|l|}{55} & 42 & \multicolumn{2}{|c|}{30} & 30 \\
\hline$\Delta$ di-6S & & 37 & \multicolumn{2}{|l|}{19} & 18 & \multicolumn{2}{|c|}{12} & 14 \\
\hline $\mathrm{II}-\mathrm{H}$ & & 40 & \multicolumn{2}{|l|}{24} & 7 & \multicolumn{2}{|c|}{31} & 18 \\
\hline \multirow[t]{2}{*}{ GlcNAc 3, 6S } & & 32 & 45 & & 25 & \multicolumn{2}{|c|}{38} & 26 \\
\hline & $\longleftarrow$ & $=\mathrm{n}$ & -pair c & s - & $\longrightarrow$ & $\longleftarrow$ & No complexes & $\longrightarrow$ \\
\hline Sulfated carbohydrate & $4 \mathrm{~K}$ & KYK & RGK & RSK & $\mathrm{KL}$ & $\mathrm{RF}$ & $\mathrm{R}$ & K \\
\hline GalNAc 4S & 22 & 22 & 11 & 11 & 18 & 2 & 0 & 0 \\
\hline$\Delta$ di-6S & 7 & 11 & 17 & 14 & 28 & 2 & 0 & 0 \\
\hline $\mathrm{II}-\mathrm{H}$ & 11 & 22 & 18 & 16 & 11 & 3 & 0 & 0 \\
\hline GIcNAc $3,6 \mathrm{~S}$ & 6 & 4 & 23 & 16 & 4 & 0 & 0 & 0 \\
\hline
\end{tabular}

*For each carbohydrate/peptide pair, a complexation affinity is reported. The peptide and carbohydrate are combined in a 7:1 ratio and analyzed by mass spectrometry. The abundance of the ion pair relative to the base peak, which is the uncomplexed peptide, is reported.

The relative complexation efficiencies for each of the carbohydrate/peptide pairs reported in Table 1 are not intended to represent quantitative binding affinities; instead the magnitude of the complexation effeciencies reported are used to classify the peptides into three broad categories: those that have a strong complexation efficiency, those with a weak complexation efficiency, and those that do not form ion-pair complexes.

All of the peptides that formed abundant ion-pair complexes (as designated in Table 1) were considered further as potentially useful ion-pairing agents. The peptides P1, P2, and P3 were quickly ruled out as ion-pairing reagents for use in the analysis of unknown sulfated carbohydrates or glycopeptides, because all three of these peptides promoted loss of sulfate in the mass spectral experiments. Loss of sulfate is a known problem in the mass spectral analysis of sulfated carbohydrates [13, 18-20], and it must be mitigated prior to compositional analysis of unknown sulfated carbohydrates. Thus, the complexing reagents that promote sulfate loss, P1, P2, and P3, are not used for identifying carbohydrates in "unknown" samples.

The two remaining peptides in the "Strong complexation effeciency" group in Table 1 were further evaluated to identify which peptide provided the strongest ion signal enhancement for the sulfated carbohydrates. In this set of experiments, an internal standard, caffeine, was added to the carbohydrate samples, and a mass spectrum was acquired before and after the ion-pairing agent was added. In the before spectrum, the signal of the carbohydrate was measured, and divided by the ion signal of the internal standard. After the peptide was added, the signal of the ion-pair complex was measured, and divided by the ion signal of the internal standard. By comparing the ion signal of the carbohydrate in the "before" spectrum with the signal of the ion-pair in the "after" spectrum, one could measure the amount of signal enhancement gained by using the ion-pairing reagents.
No matter which peptide or carbohydrate was used for these experiments, the signal for the ion-pair complex was much greater than the signal for the carbohydrate alone. This is obvious from Figure $3 a$ and $b$, respectively. The signal for the native carbohydrate (in Figure 3a) is weak, but the signal for the ion pair complex, (in Figure 3b) is strong. The ion-pairs generated with the dipeptide Lys-Lys, provided a 3- to 6-fold increase in ionization effeciency of the sulfated carbohydrates, while the ion-pairs generated with the tripeptide, Lys-Lys-Lys increased the ionization effeciency of the carbohydrates by a factor of 4 to 19; see Table 2 . Thus, while both Lys-Lys and Lys-Lys-Lys formed abundant ion-pair complexes, Lys-Lys-Lys also increased the ion signal, by up to 19 times. The enhanced efficiency of Lys-Lys-Lys is likely because it has three amino side chains, versus two for Lys-Lys. Since LysLys-Lys is effective at binding to sulfated carbohydrates and increasing the signal of the carbohydrates, it was selected as an ion-pairing reagent for sulfated glycopeptides. It is not known at this time why the tripeptide enhances the signals of the sulfated carbohydrates to a different extent, depending on the carbohydrate tested. However, based on the MS data, there is no evidence that this is due to the ion-pairing agents promoting loss of sulfate for any of the carbohydrates tested.

Table 2. Signal enhancement

\begin{tabular}{llc}
\hline Sulfated carbohydrate & $2 \mathrm{~K}$ & $3 \mathrm{~K}$ \\
\hline \hline GaINAc4S & $4^{*}$ & $19^{*}$ \\
$\Delta$ di-6S & 3 & 4 \\
II-H & 6 & 10 \\
GlcNAc 3, 6S & 3 & 13
\end{tabular}

*Signal enhancement is reported as the signal of the ion-pair complex divided by the signal of the free carbohydrate, as described in the text. The ion-pairs provide 3-19 times stronger signal compared to the native carbohydrates. 


\section{Biological Application}

Enzymatic digestion of glycoproteins, followed by mass spectral analysis of the resulting glycopeptide mixtures, has been implemented as an effective strategy to profile glycosylation in glycoproteins [16, 23-25]. (Both tryptic digests and digests using nonselective enzymes like proteinase $\mathrm{K}$ have been used for these purposes.) In such analyses, individual glycopeptides are not purified. Instead, mixtures of glycopeptides are analyzed simultaneously. While this approach is efficacious in providing site-specific glycosylation information, the neutral glycopeptides present in the mixture suppress the signal of the sulfated glycopeptides, when analysis is conducted in the positive ion mode [16].

The above method development work was executed to solve this problem by identifying an ion-pairing reagent that could enhance the signal of sulfated glycopeptides, in a complex mixture containing nonsulfated glycopeptides. To demonstrate the efficacy of this approach, the ion-pairing reagent identified above is used to characterize the sulfation in ovine luteinizing hormone, which is a glycoprotein containing a complex mixture of sulfated and nonsulfated glycoforms.

The glycoprotein, luteinizing hormone, was digested with proteinase $\mathrm{K}$ prior to analysis [16]. This digestion specifically released glycopeptides at one of the three glycosylation sites on the hormone, the $\mathrm{Asn}^{56}$ site on the $\alpha$ subunit. We have previously identified the glycopeptides obtained from this digest using MS methods [16]. While others have determined, using radiolabeling, that sulfated glycoforms are very abundant in this hormone, comprising $80 \%$ of the total carbohydrate content [9], MS data of the glycopeptides from the $\mathrm{Asn}^{56}$ site showed a strong bias towards detection of neutral glycoforms [16]. Table 3 summarizes the sulfated glycoforms characterized from radiolabeling studies. (These studies pooled glycoforms from all three glycosylation sites in the protein.) It also shows which of these glycoforms were identified by MS methods, when the glycosylation at the $\mathrm{Asn}^{56}$ site was profiled, selectively. Several of the sulfated glycoforms identified by radiolabeling were not reported in the MS assay. This may be due to the fact that these glycoforms are absent at the $\mathrm{Asn}^{56}$ site, or it may be that these glycoforms are present, but the signals for these sulfated glycopeptides were suppressed by neutral glycopeptides that dominated the MS data.

The ion-pairing reagent (Lys-Lys-Lys) is designed to facilitate the detection of sulfated glycoforms, so utilizing it should resolve whether or not the carbohydrates, (4)-(7) in Table 3, are present at the $\mathrm{Asn}^{56}$ site of luteinizing hormone. Table 4 describes each of the ion-pair complexes that could be observed when LysLys-Lys is combined with the luteinizing hormone digest, along with the expected $\mathrm{m} / \mathrm{z}$ for these complexes. The last column in the table shows which complexes were detected in the quadrupole ion trap, when LysLys-Lys is added to the glycoprotein digest. (The MS
Table 3. Sulfated glycoforms on ovine luteinizing hormone

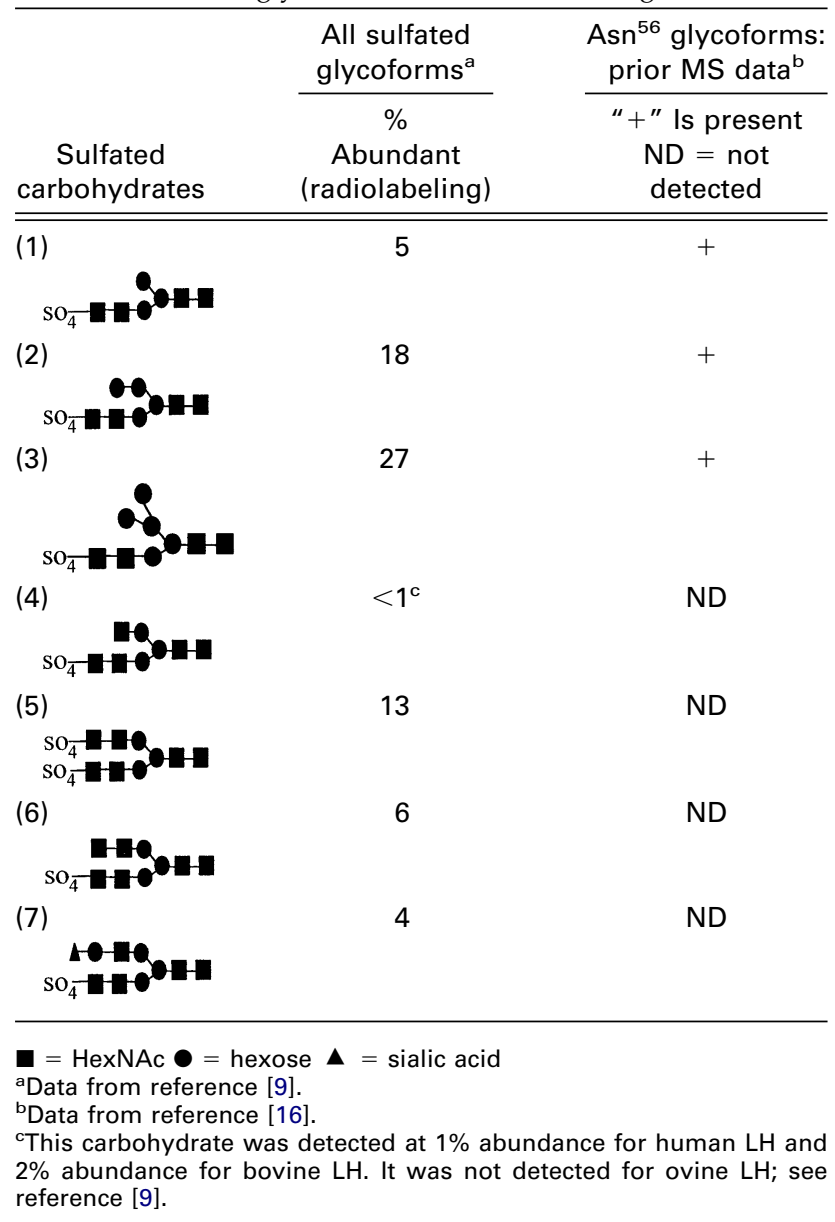

data is in Figure 4.) Five of the seven glycopeptides are detected at the $\mathrm{Asn}^{56}$ site, when the ion-pairing agent is used, including (4), which is present at very low abundance in this hormone $(<1 \%)$. Because (6) and (7) in Table 4 were not detected as ion-pair complexes, it is probable that these sulfated carbohydrates are not present at $\mathrm{Asn}^{56}$, but at another glycosylation site in this hormone. While direct MS analysis of this sample only detected sulfated glycoforms (1)-(3), incorporating the ion-pairing reagent was necessary to detect the disulfated glycopeptide, (5), and a glycopeptide present at $<1 \%$ abundance, (4).

The ion trap mass spectrum for this digest, both before and after the ion-pairing reagent is added is in Figure $4 a$ and $b$, respectively. In Figure $4 a$, the spectrum is dominated by nonsulfated glycopeptides, labeled with diamonds, that have been identified previously [16], and the signal-to-noise is mediocre. In Figure $4 b$, the nonsulfated glycorforms are much less abundant, and several new peaks are present. Each of these new peaks corresponds to the mass of a doubly charged ion-pair complex, containing a sulfated glycopeptide complexed to Lys-Lys-Lys. These peaks are labeled numerically on the mass spectrum in Figure $4 \mathrm{~b}$ to correspond with the structures described in Table 4. 
The ion trap data is useful at demonstrating that the ion-pairing method is an effective way to selectively enhance the signal of sulfated glycopeptides. The ion-pairing reagent did not form complexes with any of the nonsulfated glycopeptides present in Figure $4 \mathrm{a}$, and two new sulfated glycopeptides were identified, (4) and (5), that were not reported by previous MS assays. While a direct, quantitative comparison of the ionization efficiency of the ion-pair complexes and the neutral glycopeptides cannot be made (because quantitative data for the neutral glycoforms are not available) we estimate that the ionpair complexes enhance the detection of the sulfated glycopeptides by a factor of 5 to 10 . Unfortunately, the mass resolution and signal-to-noise are not optimal in Figure $4 \mathrm{~b}$. However, these parameters are likely attributable to the limitations of the quadrupole ion trap and not the ion-pairing method. To support the initial compositional assignments made using the ion trap data, the method was transferred to a quadrupole time-of-flight mass spectrometer.

Table 4. All possible ion-pair complexes, and those detected experimentally

\begin{tabular}{|c|c|c|}
\hline $\begin{array}{c}\text { Possible Glycopeptide } \\
\text { Adducts }\end{array}$ & Calc'd $m / z^{a}$ & $\begin{array}{c}\text { Expt'I } \mathrm{m} / \mathrm{z} \\
\text { (from QIT-MS) }\end{array}$ \\
\hline HentT + 3 & 1064.4 & 1064.5 \\
\hline (2) & 1145.5 & 1145.5 \\
\hline (3) & 1226.5 & 1226.6 \\
\hline (4) & 1166.0 & 1166.2 \\
\hline (5) & 1307.5 & 1307.7 \\
\hline (6) & 1267.5 & not detected \\
\hline (7) & 1392.5 & not detected \\
\hline
\end{tabular}

$\mathbf{\square}=$ HexNAc $\bullet=$ hexose $\boldsymbol{\Delta}$ = sialic acidNIT = the peptide Asn-Ile-Thr; $3 \mathrm{~K}=$ the ion pairing agent; Lys-Lys-Lys.

${ }^{a}$ Calculated $\mathrm{m} / \mathrm{z}^{\prime} \mathrm{s}$ are for doubly charged ions, with $\mathrm{H}+$ as the charge carrier.
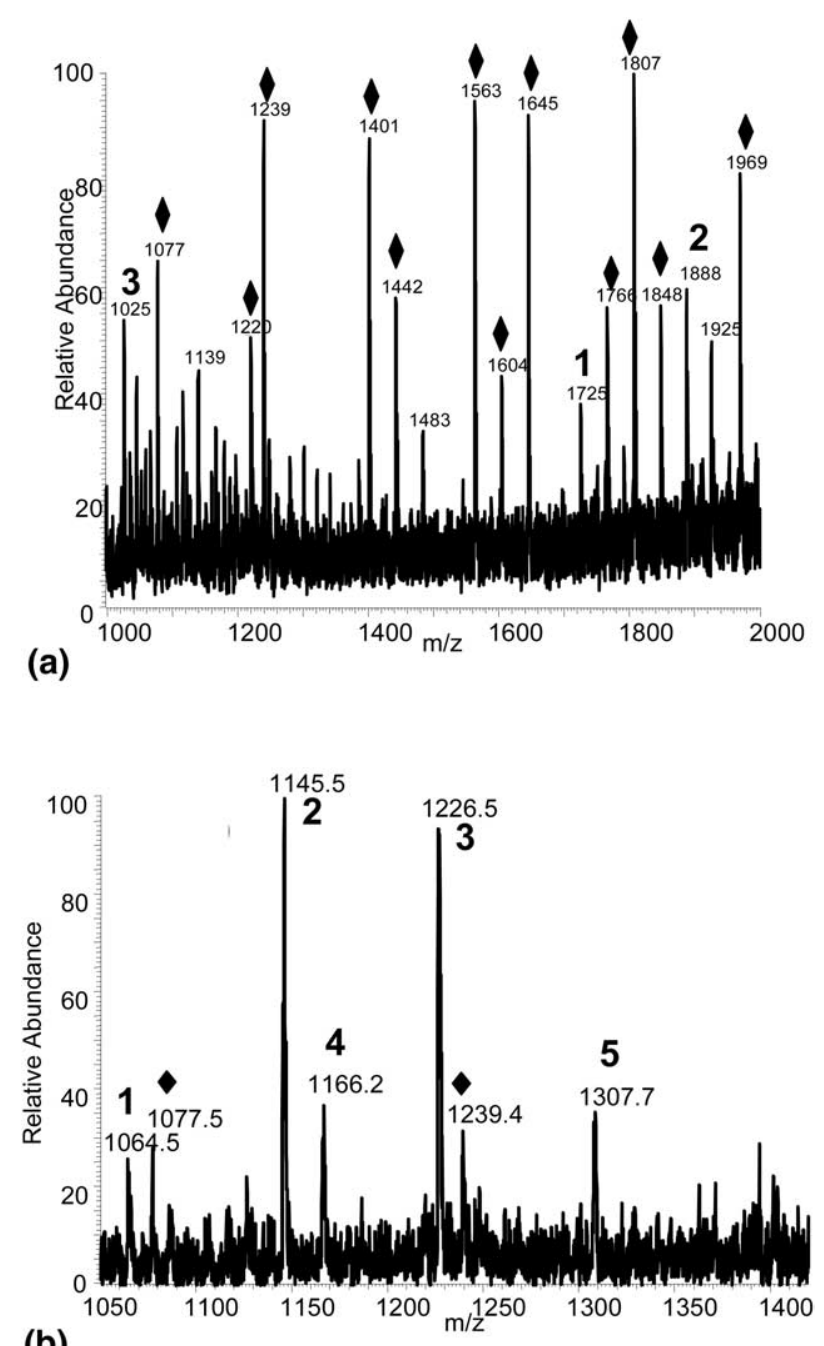

(b)

Figure 4. (a) Ion trap mass spectrum of the glycopeptide digest of oLH. Peaks with diamonds are nonsulfated glycopeptides that have been identified previously [16]; three sulfated glycopeptides (numbered 1,2, and 3 on the spectrum and described in Table 3) have also been identified[16]. (b) The glycopeptide digest in (a), with the ion-pairing agent Lys-Lys-Lys added. Ion-pair complexes containing sulfated glycopeptides are numbered 1-5 and described in Table 4.

\section{Confirmation by High Resolution Mass Spectrometry}

The qTOF data, shown in Figure 5, contain the same five ion-pair complexes identified on the ion trap. Table 5 depicts each of the structures identified by the qTOF mass spectrometer, including the nonsulfated glycoforms that were identified. The high-resolution data for each of the ions was good; the experimental mass was generally within $0.01 \mathrm{Da}$ of the theoretical mass. In addition to the high-resolution capabilities of the qTOF, this instrument is also more sensitive than the ion trap mass spectrometer, so the signal-to-noise ratio in Figure 5 is higher than in Figure 4. In contrast to the ion trap data, the qTOF data contains both $[2 \mathrm{H}+]$ or $[\mathrm{H}+$ and $\mathrm{K}+$ ] as the charge carriers for the sulfated glycopeptide 


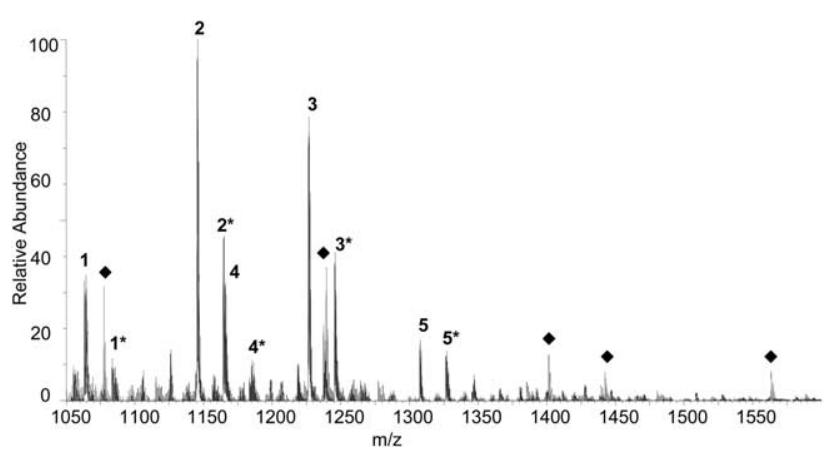

Figure 5. Q-TOF mass spectrum of the glycopeptide digest of $\mathrm{oLH}$ with the ion-pairing agent added. Peaks 1-5 are ion-pair complexes; $1^{*}-5^{*}$ are ion-pair complexes with potassium as a charge carrier; peaks with diamonds are nonsulfated glycopeptides. All labeled peaks are described in Table 5.

complexes. This leads to more peaks present in the spectrum, but their assignment is straight-forward.

\section{Additional Benefits of Ion-Pairing}

In addition to enhancing the signal of sulfated glycopeptides in positive ion ESI-MS, the ion-pairing technology described herein is useful for another reason: it facilitates structural analysis of sulfated glycopeptides. One example is shown in Figure 6; it contains MS/MS data of the ion-pair complex at $\mathrm{m} / \mathrm{z} 1145$, which is Complex (2) in Table 4. Upon collisional activation, this ion-pair complex dissociates to give many abundant product ions resulting from glycosidic cleavage. These cleavages are labeled on the spectrum using nomenclature adopted by Domon and Costello [26]. Because of the many product ions in the spectrum, the composition, sequence, and branching of this carbohydrate can be verified, as shown in Figure 6. A full investigation of the utility of ion-pairing for structural analysis of sul-

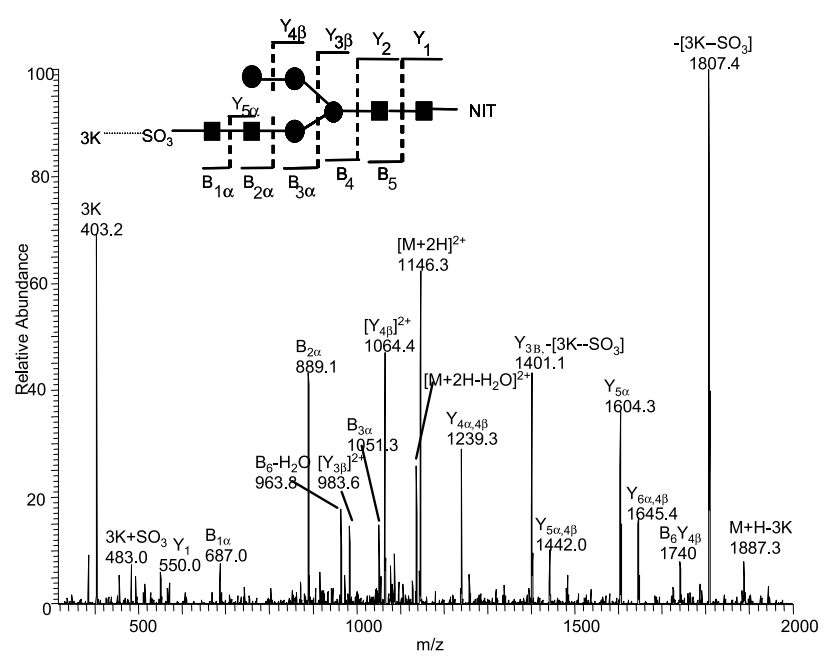

Figure 6. MS/MS data for $m / z$ 1145, Complex 2 in Table 4. This data can be used to verify the structure and composition of the glycopeptide.

fated glycopeptides is ongoing and will be presented separately.

\section{Comparision to (-)ESI-MS}

While it is well-known that sulfated carbohydrates are detected and characterized using negative ion ESI-MS $[6-8,13]$, this detection method is not ideal for sulfated glycopeptides. Figure 7 displays MS data demonstrating this. When the glycopeptide mixture in Figure $4 \mathrm{a}$ is ionized by negative ion mode, the MS data in Figure 7a is obtained. Four peaks appear in the spectrum that are consistent with the $\mathrm{m} / \mathrm{z}$ values expected for doubly deprotonated, sulfated glycopeptides (2), (3), (4), and (5). The glycopeptide (1) was not detected in the negative ion mode, perhaps because the ion signal is low. (It is somewhat surprising that the negative ion signal of

Table 5. Glycopeptides identified in qTOF data

\begin{tabular}{|c|c|c|c|c|}
\hline lons identified & Charge carrier & Calc'd m/z & Expt'I m/z & Mass diff \\
\hline$\overline{(1)^{1}}$ & $2 \mathrm{H}^{+}$ & 1064.47 & 1064.42 & $0.050^{2}$ \\
\hline$(\mathrm{Hex})(\mathrm{HexNAc})_{2}-\mathrm{NIT}^{3}$ & $\mathrm{H}^{+}$ & 1077.46 & 1077.45 & 0.011 \\
\hline (1) $\left(\mathrm{K}^{+}\right.$adduct $)$ & $\mathrm{H}^{+}$and $\mathrm{K}^{+}$ & 1083.44 & 1083.44 & 0.004 \\
\hline (2) & $2 \mathrm{H}^{+}$ & 1145.49 & 1145.48 & 0.008 \\
\hline (2) $\left(\mathrm{K}^{+}\right.$adduct $)$ & $\mathrm{H}^{+}$and $\mathrm{K}^{+}$ & 1164.47 & 1164.46 & 0.008 \\
\hline (4) & $2 \mathrm{H}^{+}$ & 1166.00 & 1165.98 & $0.024^{2}$ \\
\hline (4) $\left(\mathrm{K}^{+}\right.$adduct $)$ & $\mathrm{H}^{+}$and $\mathrm{K}^{+}$ & 1184.98 & 1184.97 & 0.013 \\
\hline (3) & $2 \mathrm{H}^{+}$ & 1226.52 & 1226.52 & 0.002 \\
\hline$(\mathrm{Hex})_{3}(\mathrm{HexNAc})_{2}-\mathrm{NIT}^{3}$ & $\mathrm{H}^{+}$ & 1239.51 & 1239.51 & 0.003 \\
\hline (3) $\left(\mathrm{K}^{+}\right.$adduct $)$ & $\mathrm{H}^{+}$and $\mathrm{K}^{+}$ & 1245.49 & 1245.49 & 0.004 \\
\hline (5) & $2 \mathrm{H}^{+}$ & 1307.52 & 1307.52 & -0.003 \\
\hline (5) $\left(\mathrm{K}^{+}\right.$adduct $)$ & $\mathrm{H}^{+}$and $\mathrm{K}^{+}$ & 1326.50 & 1326.50 & 0.001 \\
\hline$(\mathrm{Hex})_{4}(\mathrm{HexNAc})_{2}-\mathrm{NIT}^{3}$ & $\mathrm{H}^{+}$ & 1401.56 & 1401.57 & -0.003 \\
\hline$(\mathrm{Hex})_{3}(\mathrm{HexNAc})_{3}-\mathrm{NIT}^{3}$ & $\mathrm{H}^{+}$ & 1442.59 & 1442.59 & 0.003 \\
\hline$(\mathrm{Hex})_{5}(\mathrm{HexNAc})_{2}-\mathrm{NIT}^{3}$ & $\mathrm{H}^{+}$ & 1563.62 & 1563.62 & -0.005 \\
\hline
\end{tabular}

${ }^{1}$ Parenthetical numbers refer to the sulfated glycopeptides described in Table 4.

${ }^{2}$ Mass accuracy compromised due to isobaric interference.

${ }^{3}$ Nonsulfated glycopeptides were identified previously in reference [16]. 

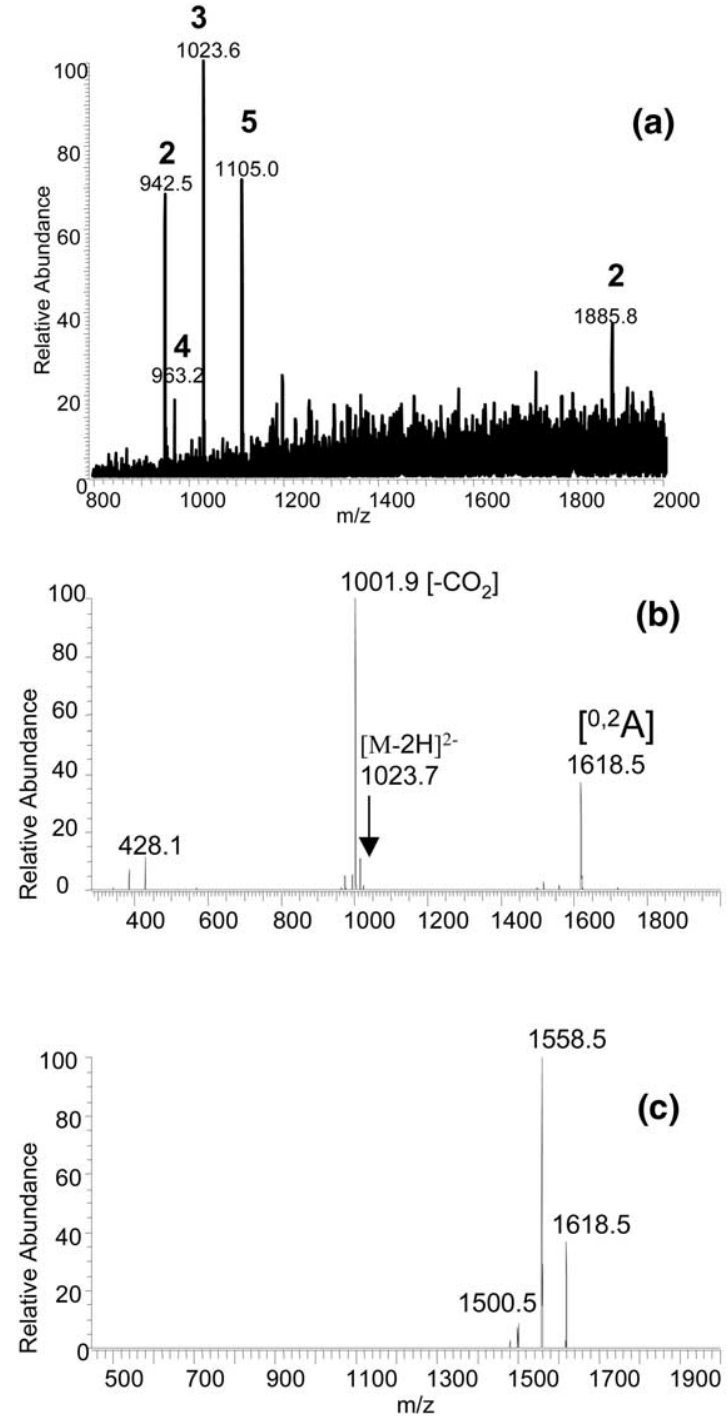

Figure 7. (a) Mass spectrum of the glycopeptide digest, ionized in negative ion mode. The labeled peaks are consistent with the expected $\mathrm{m} / \mathrm{z}$ for sulfated glycopeptides (2)-(5). (b) MS/MS data for $m / z$ 1023.7. While this mass is consistent with that of a doubly charged, sulfated glycopeptide, the MS/MS data does not confirm the ion's composition. (c) MS ${ }^{3}$ data $(1023.7 \rightarrow 1816 \rightarrow)$. Again, the spectrum is not consistent with that of a sulfated carbohydrate.

glycopeptides is weak. Perhaps the basic amino groups on the peptide, which are generally protonated in solution, suppress the negative signal to some extent.)

The (-)ESI-MS data is less useful than the ionpairing data for other reasons as well. MS ${ }^{\mathrm{n}}$ experiments on the doubly deprotonated ions in Figure 7a, cannot be used to substantiate the composition assignment of these ions. As an example, $\mathrm{MS}^{2}$ and $\mathrm{MS}^{3}$ data for $\mathrm{m} / \mathrm{z}$ 1023.6 is in Figure $7 \mathrm{~b}$ and c, respectively. (The MS ${ }^{\mathrm{n}}$ data for the other doubly charged ions in Figure 7a were all very similar to the data in $7 \mathrm{~b}$ and $7 \mathrm{c}$; so Figure $7 \mathrm{~b}$ and $c$ are representatives examples of CID data for all these ions.) The $\mathrm{MS}^{\mathrm{n}}$ data appears to support the claim that these peaks are not sulfated glycopeptides. None of the product ions typically abundant in MS/MS data of sulfated carbohydrates are observed. Loss of sulfate is not observed. No glycosidic cleavage ions are detected either, which are typically very abundant in CID spectra of carbohydrates $[7,8,13]$. Instead, an apparent loss of $\mathrm{CO}_{2}$ is the base peak in the MS/MS data, and the only other prominent ions in the MS/MS data are a pair of complimentary, singly charged ions, $\mathrm{m} / \mathrm{z} 428$ and 1618 . (These ions combine to produce the doubly charged parent, $m / z$ 1023.7.) The pair of ions does not correspond to cleavage at a glycosidic or peptide bond. Assuming this MS/MS spectrum is, in fact, from glycopeptide (2), it is mathematically possible that these ions are the result of a ${ }^{0,2} \mathrm{~A}$ cross-ring cleavage; however, an MS $^{3}$ experiment on this ion (in Figure 7c) is not consistent with typical glycan fragmentation either.

This data is clearly very different than previously published negative ion data for sulfated glycans and glycolipids. These differences can be attributed to the fact that the sulfated glycoconjugates in this study are glycopeptides, and the peptide portion drives the fragmentation. This reasoning is supported by the fact that loss of $\mathrm{CO}_{2}$ is the base peak in the MS/MS spectrum. This loss had to come from the peptide, as there are no carboxylate groups in the carbohydrate.

In summary, the lack of glycosidic cleavages, peptide cleavages, or a loss of $\mathrm{SO}_{3}$ in the (-)ESI-MS ${ }^{\mathrm{n}}$ spectra of this compound provide no substantiating evidence that this ion is, in fact, the sulfated glycopeptide in question. By contrast, the ion-pairing technique enhanced the signal of the sulfated glycopeptides in positive ion mode, and MS/MS data of the ion-pair complex could be used to confirm the composition and the structure of the ions.

\section{Conclusion}

By screening a small library of 13 compounds as potential ion-pairing reagents for sulfated carbohydrates, several small peptides were identified that coordinated to sulfated carbohydrates and produced strong ion-pair complexes, observable by mass spectrometry. Upon further evaluation, the peptide Lys-Lys-Lys was deemed an optimal ion-pairing reagent for enhancing the signal of sulfated carbohydrates. When it is added to a mixture of glycopeptides originating from luteinizing hormone, the sulfated glycoforms that are present readily form ion-pair complexes with Lys-Lys-Lys. While the mass spectral signal of sulfated glycoforms is typically suppressed in positive ion mode, when nonsulfated glycoforms are present, the ion-pairing reagent selectively enhanced the signal of the sulfated species. Using this technique, a disulfated glycoform, previously not detected by MS methods, is identified, and a sulfated glycoform that is present in very low abundance ( $<1 \%$ based on radiolabeling assays) is detected, without any separation or enrichment of these species prior to analysis. Finally, this method appears very promising for the structural analysis of sulfated glyco- 
peptides, and it is advantageous to detection of sulfated glycopeptides in the negative ion mode.

\section{Acknowledgments}

The authors acknowledge the NIH for funding (project number 1 P20 RR17708-01) and Dr. George Bousfield of Wichita State University for supplying the ovine luteinizing hormone glycopeptides.

\section{References}

1. Ernst, S.; Langer, R.; Cooney, C.; Sasisekharan, R. Enzymatic degradation of glycosaminoglycans. Crit. Rev. Biochem. Mol. Biol. 1995, 30(5), 387-444.

2. Plaas, A. H. K.; West, L. A.; Wong-Palms, S.; Nelson, F. R. T. Glycosaminoglycan sulfation in human osteoarthritis. J. Biol. Chem. 1998, 273(20), 12642-12649.

3. Bayliss, M. T.; Osborne, D.; Woodhouse, S.; Davidson, C. Sulfation of chondroitin sulfate in human articular cartilage. J. Biol. Chem. 1999, 274(22), 15892-15900.

4. Desaire, H.; Sirich, T. L.; Leary, J. A. Evidence for block and randomly-sequenced chondroitin polysaccharides: Sequential enzymatic digestion and quantification using ion trap tandem mass spectrometry. Anal. Chem. 2001, 73(15), 3513-3520.

5. Bowman, K. G.; Cook, B. N.; de Graffenried, C. L.; Bertozzi, C. R. Biosynthesis of L-selectin ligands: Sulfation of sialyl Lewis x-related oligosaccharides by a family of GlcNAc-6-sulfotransferases. Biochemistry 2001, 40, 5382-5391.

6. Kawasaki, N.; Haishima, Y.; Ohta, M.; Satsuki, I.; Hyuga, M.; Hyuga, S.; Hayakawa, T. Structural analysis of sulfated $\mathrm{N}$ linked oligosaccharides in erythropoietin. Glycobiology 2001, 11(12), 1043-1049.

7. Thomsson, K. A.; Karlsson, H.; Hansson, G. C. Sequencing of sulfated oligosaccharides from mucins by liquid chromatography and electrospray ionization tandem mass spectrometry. Anal. Chem. 2000, 72, 4543-4539.

8. Thomsson, K. A.; Karlsson, N. G.; Hansson, G. C. Liquid chromatography-electrospray mass spectrometry as a tool for the analysis of sulfated oligosaccharides from mucin glycoproteins. J. Chromatogr. A. 1999, 854, 131-139.

9. Green, E. D.; Baenziger, J. U. Asparagine-linked oligosaccharides on lutropin, follitropin, and thyrotropin. J. Biol. Chem. 1988, 26(1), 36-44.

10. Hemmerich, S.; Rosen, S. D. Carbohydrate sulfotransferases in lymphocyte homing. Glycoboiology 2000, 10(9), 849-856.

11. Bai, X.; Brown, J. R.; Varki, A.; Esko, J. D. Enhanced 3-Osulfation of galactose in Asn-linked glycans and Maackia amurenesis lectin binding in a new Chinese hamster ovary cell line. Glycobiology 2001, 11(8), 621-632.

12. Fiete, D.; Srivastava, V.; Hindsgau, O.; Baenziger, J. U. A hepatic reticuloendothel cell receptor specific for $\mathrm{SO}_{4}-4$ GalNAc $\beta \quad 1,4$ GlcNAc $\beta$ 1,2Man $\alpha$ that mediates rapid clearance of lutropin. Cell 1991, 67, 1103-1110.
13. Zaia, J. Mass spectrometry of oligosaccharides. Mass Spectrom. Rev. 2004, 23, 161-277.

14. De Graffenreid, C. L.; Bertozzi, C. R. Golgi localization of carbohydrate sulfotransferases is a determinant of L-selectin ligand biosynthesis. J. Biol. Chem. 2003, 278(41), 40282-40295.

15. Delcommenne, M.; Kannagi, R.; Johnson, P. TNF-a increases the carbohydrate sulfation of CD44: induction of 6-sulfo $\mathrm{N}$-acetyllactosamine on N- and O-linked glycans. Glycobiology 2002, 12(10), 613-622.

16. Jiang, H.; Butnev, V. Y.; Bousfield, G. R.; Desaire, H. Glycoprotein profiling by electrospray mass spectrometry. J. Am Soc. Mass Spectrom. 2004, 15, 750-758.

17. Juhasz, P.; Biemann, K. Mass-spectrometric molecular weight determination of highly acidic compounds of biological significance via their complexes with basic polypeptides. Proc. Natl. Acad. Sci. U.S.A. 1994, 91, 4333.

18. Siegel, M. M.; Tabei, K.; Kagan, M. Z.; Vlahov, I. R.; Hileman R. E.; Linhardt, R. J. Polysulfated carbohydrates analyzed as ion-paired complexes with basic peptides and proteins using electrospray negative ionization mass spectrometry. J. Mass Spectrom. 1997, 32, 760-772.

19. Gunay, N. S.; Tadano-Aritomi, K.; Toida, T.; Ishizuka, I.; Linhardt, R. J. Evaluation of counterions for electrospray ionization mass spectral sulfated analysis of a highly sulfated carbohydrate, sucrose octasulfate. Anal. Chem. 2003, 75(13), 3226-3231.

20. Kuberan, R.; Lech, M.; Zhang, L.; Wu, Z. L.; Beeler, D. L.; Rosenberg, R. D. Analysis of heparan sulfate oligosaccharides with ion pair reverse phase capillary high performance liquid chromatography-microelectrospray ionization time-of-flight mass spectrometry. J. Am. Chem. Soc. 2002, 124, 8707-8718.

21. Desaire, H.; Leary, J. A. The effects of coordination number and ligand size on the gas phase dissociation and stereochemical differentiation of co-coordinated monosaccharides. Int. J. Mass Spectrom. 2001, 209, 171-184.

22. Desaire, H.; Leavell, M. D.; Leary, J. A. Solvent effects in tandem mass spectrometry: Mechanistic studies indicating how a change in solvent conditions and $\mathrm{pH}$ can dramatically alter CID spectra. J. Org. Chem. 2002, 67(11), 3693-3699.

23. An, H. J.; Peavy, T. R.; Hedrick, J. L.; Lebrilla, C. B. Determination of $\mathrm{N}$-glycosylation sites and site heterogeneity in glycoproteins. Anal. Chem. 2003, 75, 5628-5673.

24. Zhu, X.; Borchers, C.; Bienstock, R. J.; Tomer, K. B. Mass spectrometric characterization of glycosylation pattern of HIV-gp120 expressed in CHO cells. Biochemistry 2000, 39, 11194-11204.

25. Juhasz, P.; Martin, S. A. The utility of nonspecific proteases in the characterization of glycoproteins by high-resolution timeof-flight mass spectrometry. Int. J. Mass Spectrom. 1997, 169/ $170,217-230$

26. Domon, B.; Costello, C. E. A systematic nomenclature for carbohydrate fragmentations in FAB-MS/MS spectra of glycoconjugates. Glycoconj. J. 1988, 5, 397-409. 